\section{$\underset{\substack{\text { hommes } \\ \text { \& migrations }}}{ }$}

\section{Hommes \& migrations}

Revue française de référence sur les dynamiques

migratoires

$1313 \mid 2016$

1983 , le tournant médiatique

\title{
Les O. S. immigrés à l'écran
}

Les luttes de la « première génération » lors des conflits de l'automobile (1981-1984)

\section{Stéphane Kronenberger}

\section{(2) OpenEdition}

\section{Journals}

Édition électronique

URL : http://journals.openedition.org/hommesmigrations/3559

DOI : 10.4000/hommesmigrations.3559

ISSN : 2262-3353

Éditeur

Musée national de l'histoire de l'immigration

Édition imprimée

Date de publication : 1 janvier 2016

Pagination : 45-53

ISBN : 978-2-919040-34-6

ISSN : $1142-852 X$

Référence électronique

Stéphane Kronenberger, « Les O. S. immigrés à l'écran », Hommes \& migrations [En ligne], 1313 | 2016, mis en ligne le 01 janvier 2019, consulté le 16 mars 2020. URL : http://journals.openedition.org/ hommesmigrations/3559; DOI : https://doi.org/10.4000/hommesmigrations.3559 


\title{
LES OS IMMIGRÉS À L'ÉCRAN
}

\section{LES LUTTES DE LA « PREMIĖRE GÉNÉRATION » LORS DES CONFLITS DE L'AUTOMOBILE (1981-1984)}

par STÉPHANE KRONENBERGER, Post-doctorant à l'université d'Aix-Marseille (TELEMME).

\author{
Des grèves éclatent dans l'industrie automobile dès l'automne \\ 1981, mais I'année 1983 constitue un tournant marqué par \\ des conflits à Renault Flins, Citroën Aulnay et surtout Talbot \\ Poissy. L'analyse des images de la couverture télévisuelle \\ de l'événement met en exergue la volonté de la «première \\ génération » des OS immigrés de revendiquer son droit \\ à la dignité et à l'égalité. Mais l'adoption d'une politique \\ économique de rigueur et l'attitude ambiguë de certaines \\ centrales syndicales font voler en éclats ces aspirations \\ légitimes à l'intégration dans la société française.
}

Après la Seconde Guerre mondiale, l'industrie automobile, où le taylorisme règne désormais en maître, emploie de nombreux OS immigrés. Recrutés en grande partie au Maroc, mais aussi dans les autres pays du Maghreb ou en Turquie, ces paysans analphabètes constituent une main-d'œuvre corvéable à merci à laquelle sont confiés les travaux pénibles et les tâches répétitives ne requérant presque aucune qualification. Or, depuis le premier choc pétrolier de 1973, la crise s'installe et se traduit dans ce secteur par un phénomène de concentration provoque de nombreuses suppressions d'emplois. Entré au capital de Citroën en 1974, Peugeot rachète définitivement l'entreprise en 1976, et fait de même deux ans plus tard avec Chrysler France (ex-Simca) bientôt rebaptisée Talbot. Ces acquisitions font notamment entrer dans son giron les usines de Poissy ${ }^{1}$ et Aulnay employant au moins une moitié d'étrangers, dont les trois quarts des ouvriers travaillent à la chaîne. Le second choc pétrolier, consécutif à la révolution iranienne de 1979, aggrave encore la situation. Dès 1977, une aide au retour de 10000 francs est d'ailleurs proposée par le secrétaire d'État chargé des travailleurs manuels et immigrés, Lionel Stoléru, mais ce "million » ne rencontre pas le succès escompté, et la mesure est finalement abolie à la suite de l'élection de François Mitterrand.

À peine six mois après l'arrivée de la gauche au pouvoir, des grèves éclatent à l'automne 1981 
dans le fief de Peugeot à Sochaux ${ }^{2}$ mais aussi chez Renault à Billancourt et à Sandouville. Ces mouvements sociaux se propagent au mois d'avril 1982 à une autre usine de la régie à Flins, puis à Citroën Aulnay entre le 23 avril et le $1^{\text {er }}$ juin, et enfin à Talbot Poissy en juin et début juillet. Après s'être longtemps désintéressés de la contribution des travailleurs immigrés de la "première génération " à la bonne marche de l'économie française, les journaux télévisés leur offrent, lors de ces grèves, une exposition médiatique maximale, dont il est intéressant de comprendre les enjeux. Comment les reportages télévisés en particulier rendent-ils compte des aspirations de ces prolétaires venus d'ailleurs et du rapport qu'ils entretiennent avec une société française au sein de laquelle ils vivent depuis de nombreuses années? Au diapason des autres médias, la télévision est une caisse de résonance de représentations négatives à propos des travailleurs immigrés : les

Au sein de ces " usines

de la peur ", l'expression des travailleurs est, en effet, de longue date confisquée par un syndicat dit « indépendant », la Confédération des syndicats libres (CSL), foncièrement anticommuniste et inféodé au patronat. hommes de cette génération usés et sans ressort sont ainsi abandonnés à leur triste sort après avoir été quelques années plus tôt victimes d'un racisme virulent. Contrairement aux grèves du début des années 1970 chez Renault $^{3}$ durant lesquelles les journalistes demeurent cantonnés aux portes des usines ${ }^{4}$, les luttes se déroulant une décennie plus tard sont l'occasion de rentrer à l'intérieur des ateliers et de filmer les immigrés dans leur labeur quotidien sur les chaînes d'assemblage, mais aussi de se faire, auprès de l'opinion, le relais de leurs aspirations. Cela est d'autant plus intéressant que ces individus se trouvent alors, après une décennie passée en France, voire davantage, à un moment charnière, tant dans leur itinéraire professionnel que dans leur vie personnelle.

\section{Des grèves calmes et tranquilles ?}

Les images filmées en janvier 1983 à Renault Flins laissent transparaître le caractère bon enfant de l'atmosphère régnant au sein de l'usine malgré la grève. Rendre compte de ce climat de dialogue entre la base, composée majoritairement d'ouvriers immigrés, et les agents de maîtrise ou cadres français, voire des taquineries réciproques des uns envers les autres, est loin d'être anodin ${ }^{5}$. Il s'agit de marquer le contraste avec les scènes d'affrontements physiques lors de certaines grèves de l'année précédente. Ainsi, le 3 juin 1982 se sont déroulés à l'usine Talbot de Poissy des affrontements assez violents, dont le bilan atteint une quarantaine de blessés. Ces incidents font d'ailleurs le soir même l'ouverture du journal télévisé de 20 heures et ont provoqué quelques invectives verbales à l'Assemblée nationale ${ }^{6}$. Des salariés non grévistes, membres du syndicat maison la Confédération des syndicats libres (CSL ${ }^{7}$ ), emmenés par le chef du personnel, ont vers 17 h 30 tenté de déloger de l'atelier qu'ils occupaient les travailleurs immigrés soutenus par la CGT. Ces derniers avaient cessé le travail pour réclamer, comme précédemment chez Citroën, certes des augmentations de salaire et l'amélioration de leur vie d'OS, mais aussi le respect des libertés syndicales et la fin des humiliations quotidiennes dans les rapports avec l'encadrement, en un mot le respect de leur « dignité ». Au sein de ces « usines de la peur ${ }^{8}$ », l'expression des travailleurs est, en effet, de longue date confisquée par un syndicat dit « indépendant », la Confédération des syndicats libres (CSL), foncièrement anticommuniste et inféodé au patronat. La carte d'adhésion est ainsi proposée dès l'embauche et il est dans l'intérêt de l'ouvrier de l'accepter s'il désire obtenir un logement ou de l'avancement. Ce corporatisme autoritaire, mêlant carotte et bâton, 
se fissure cependant largement, puisque la CFDT et surtout la CGT progressent à grands pas lors des élections professionnelles, et le syndicat dirigé par Henri Krasucki accroît fortement, à partir de 1982, le nombre de ses adhérents parmi les OS immigrés de l'automobile 9 .

\section{Les espoirs d'une intégration à la classe ouvrière française (janvier-juin 1983)}

Face à ce "mai 68 des travailleurs immigrés », le ministre du Travail nomme comme médiateur le professeur de droit social Jean-Jacques Dupeyroux. Parvenu à faire cesser pour un temps les mouvements revendicatifs à Citroën Aulnay, puis chez Talbot Poissy, il promeut la mise en place de structures de dialogue et de concertation. Ces commissions sont chargées d'étudier la question des salaires, mais aussi les possibilités existantes en termes d'évolution vers le haut des carrières des OS de l'industrie automobile ${ }^{10}$. Entre l'été et l'automne 1982, Jean Auroux fait parallèlement adopter par le Parlement des lois modifiant profondément le code du travail et favorisant notamment la vie et l'expression des salariés dans l'entreprise. Ces grèves de l'automobile coïncident donc avec un premier aboutissement des luttes engagées près d'une décennie plus tôt à Aulnay ou Poissy par quelques syndicalistes et une poignée de militants courageux ayant pris clandestinement leur carte à la CGT ou à la CFDT, au risque de se faire passer à tabac par les nervis de la CSL en cas de dénonciation.

Sur le plan médiatique, le vent de liberté qui commence à souffler dans ces usines se traduit par l'apparition à la tête des ouvriers en grève, mais aussi face à la caméra, de figures nouvelles, à l'instar de Nora Tréhel la dirigeante de la CGT à l'usine Talbot de Poissy ${ }^{11}$. Son point de vue a d'autant plus de résonance et de pertinence que les reportages proposent souvent en contrepoint au téléspectateur les arguments simplistes et caricaturaux de représentants de la CSL sur la nécessité du retour à l'ordre et le rétablissement de la liberté du travail. À Citroën Aulnay, un autre syndicaliste s'impose à l'écran en la personne d'Akka Ghazi. Après avoir dû prendre sa carte à la CSL durant une décennie, cet ouvrier marocain, fils de paysan, rejoint la CGT et devient dès 1982 à l'égard de l'opinion le porte-voix des OS en lutte pour leur dignité $^{12}$. Son aura médiatique se renforce en février de l'année suivante à l'occasion de mises à pied conservatoires décidées à son encontre et celle d'une trentaine de ses camarades $^{13}$. Il se retrouve d'ail-
Sur le plan médiatique, le vent de liberté qui commence à souffler dans ces usines se traduit par l'apparition à la tête des ouvriers en grève, mais aussi face à la caméra, de figures nouvelles, à l'instar de Nora Tréhel la dirigeante de la CGT à l'usine Talbot de Poissy. leurs, le 20 mars, soit à peine cinq semaines plus tard, sur le plateau de l'émission phare de TF1 Sept sur sept, où il continue à interpréter à la perfection son rôle de « travailleur parmi les travailleurs » pour le plus grand plaisir de ses interlocuteurs. Ces derniers s'interdisent certes de mettre en exergue le conflit en cours, mais font néanmoins réagir leur invité sur la montée du racisme à l'égard des immigrés ${ }^{14}$. À son image de leader charismitique se mêle également celle d'un personnage énigmatique tentant de concilier défense des droits des travailleurs et maintien du lien avec le roi du Maroc, qui ne voit pourtant pas d'un bon œil cette agitation sociale, dont certains de ses sujets constituent l'avant-garde.

\section{Dignité et égalité}

Les journalistes dépêchés dans les usines tentent également de rendre compte de la soif d'expression des ouvriers eux-mêmes. La liberté de donner son 
avis fait, en effet, partie des acquis les plus importants de l'année 1982. Lors de la grève des peintres de l'usine Renault de Flins est, par exemple, longuement interviewé, en janvier 1983, sur le lieu même de son labeur quotidien mais en habits civils, Taleb Ould Mohamed, OS de 32 ans qui travaille à la régie déjà depuis 1969. Il considère d'ailleurs cet endroit comme sa "maison personnelle », qui lui permet de faire vivre, avec son modeste salaire de 6000 francs primes comprises, sa mère, sa belle-mère, sa femme et ses 4 enfants ${ }^{15}$. On est loin de l'immigré célibataire auquel le journaliste demande s'il envisage de rentrer au pays. La figure choisie est, au contraire, celle d'un individu s'exprimant aisément en français et désirant ferme-
Le reportage diffusé

par Antenne 2 dès son édition

de la mi-journée montre

ainsi parfaitement que

ce face-à-face a pris les traits

d'un véritable affrontement

racial. ment rester dans l'Hexagone. Il a d'ailleurs profité du regroupement familial pour atténuer les liens le rattachant à l'autre rive de la Méditerranée. L'OS peut alors exprimer son droit de bénéficier de meilleures

conditions de travail, en décrivant les affres subits au quotidien par lui et ses collègues dans l'enfer que constituent les archaïques cabines de peinture de l'usine de Flins.

Cette aspiration à la dignité se double d'un désir légitime d'égalité, afin de pouvoir construire un meilleur avenir pour lui et sa famille. En témoigne d'abord la demande de refonte de la grille des rémunérations. Dans cette droite ligne est ensuite exprimée la nécessité de l'accès à la formation continue pour permettre à ceux qui en ont la volonté et les capacités de sortir par le haut du carcan de la condition d'OS et donc au final de se fondre dans la classe ouvrière française. Contrairement aux images associées à la grève des loyers survenue dans les foyers Sonacotra quelques années auparavant, cette séquence télévisuelle met en exergue le fait qu'il s'agirait moins d'un conflit d'immigrés que d'une revendication collective transcendant les appartenances nationales. Cet élément est réaffirmé par le protagoniste principal en excipit de l'interview, lorsqu'il indique que les huit Français de l'atelier ont également cessé le travail. Le doute sur ce point précis tente néanmoins d'être instillé dans les esprits par le pouvoir socialiste.

\section{L'islam en question}

En 1982 à Citroën Aulnay, "le déroulement et les lieux de la grève rendent visibles les pratiques religieuses des ouvriers ${ }^{16}$ ». Lorsque la prière s'effectue sur le parking et non plus dans l'atelier, elle a toutes les chances d'être filmée par les caméras de télévision. La demande de lieux de prière dignes de ce nom fait, en outre, partie des revendications. Lorsqu'un nouveau conflit éclate dans la même usine dès le début de l'année suivante, le gouvernement de gauche n'hésite pas à faire peser sur les revendications des OS immigrés la suspicion de la manipulation religieuse, pour tenter in fine de discréditer, aux yeux de l'opinion, ces mouvements sociaux à répétition. Le Premier ministre Pierre Mauroy déclare ainsi en janvier 1983 que « des travailleurs immigrés sont agités par des groupes religieux et politiques qui se déterminent en fonction de critères ayant peu à voir avec les réalités sociales françaises $^{17}$ ». Le ministre de l'Intérieur, Gaston Deferre, parle lui sciemment « de chiites ${ }^{18}$ » et « d'intégristes » alors que celui du Travail, Jean Auroux, évoque entre autres des « pratiques terroristes ». Les journaux télévisés diffusent tous sensiblement la même scène d'un groupe d'OS immigrés entourant un homme barbu ayant à la main un micro pour les haranguer en langue arabe. Mais les journalistes se rendent aussi à Aulnay pour recueillir les sentiments des uns et des autres sur les déclarations gouvernementales. Un syndicaliste évoque l'existence de « marabouts », de « hadj », qui demandent

15. "Immigrés Renault Flins ", JT de 13 heures d’Antenne 2 du 28 janvier 1983. 16. Vincent Gay, "Grèves saintes ou grèves ouvrières? Le "problème musulman" dans les conflits de l'automobile 1982-1983 ", in Genèses, n 98, mars 2015 p. 114. 17. Le Monde des 29 et 31 janvier 1983 citant une interview donnée par le Premier ministre au quotidien Nord-Éclair. 18. Le chiisme est la religion d'État en Iran, alors que les Maghrébins et les Turcs sont très majoritairement d’obédience sunnite. 
le " marronnier » annuel proposé sur les antennes nationales ou régionales à l'occasion du ramadan, on est ainsi passé d'un reportage où une femme, gardienne des traditions, explique la raison de la célébration de l'Aïd el-Kébir à des scènes de prière collective et masculine, voire à l'égorgement du mouton ${ }^{21}$. Néanmoins, six ans avant l'affaire du voile de Creil, les médias choisissent encore de traiter de situations extérieures à l'Hexagone pour illustrer le phénomène du fondamentalisme musulman, comme en témoigne le lancement de l'émission hebdomadaire Sept sur sept du 6 février 1983, soit quelques jours après les déclarations de Gaston Deferre : "Voilà ce que nous avons retenu pour vous dans l'actualité de cette semaine. D'abord le débat sur les ouvriers maghrébins dans les usines d'automobiles qui seraient manipulés par des intégristes musulmans. Nous vous expliquerons ce qu'est l'intégrisme dans un pays arabe très proche de nous et récemment touché par ce phénomène, la Tunisie ${ }^{22}$.»

Sortie d'usine Talbot-Poissy. (๐ AGENCE IM'MÉDIA.

à des travailleurs de mettre la main sur le coran et de jurer fidélité à l'organisation syndicale, sousentendu la CGT. Akka Ghazi, lui-même parfois présenté comme "l'Ayatollah d'Aulnay ", s'inscrit en faux contre cette prétendue manipulation et précise que la seule présence à caractère religieux au sein de l'usine est constituée par des locaux pour la prière obtenus récemment et utilisés par une partie seulement du personnel ${ }^{19}$.

Pour les journalistes, l'islam demeure encore un enjeu mineur, même si les représentations qui lui sont associées sur les écrans ont été fortement modifiées à la suite des images diffusées en boucle lors de la révolution iranienne ${ }^{20}$. Pour

\section{La « disparition » des ouvriers immigrés (juillet 1983-janvier 1984)}

Dans la seconde moitié de l'année 1983, les caméras saisissent une sorte de descente aux enfers effective et symbolique de cette "première génération " d'OS immigrés pour lesquels les portes se referment les unes après les autres, ce qui ne semble leur laisser au final comme alternatives que le chômage ou le retour au pays.

Lors du comité central d'entreprise du 21 juillet 1983, PSA annonce plus de 7000 suppressions d'emplois. Des débrayages spontanés ont alors lieu dans différentes usines du groupe, notamment à

19. "Situation Citroën ", JT d'Antenne 2 de 20 heures du 3 février 1983. 20. Thomas Deltombe, L'Islam imaginaire. La construction médiatique de l'islamophobie en France 1975-2005, Paris, La Découverte, 2005. 21. Édouard Mills-Affif, Filmer les immigrés. Les représentations audiovisuelles de l'immigration à la télévision française 1960-1986, Bruxelles, De Boeck, 2004, p. 250 et ss; Stéphane Kronenberger, "Les écrans de l'immigration en Franche-Comté ", in Migrations Société, vol. 26 n 151 , janvier-février 2014, pp. 105-123. 22. 7/7 du 6 février 1983. 23. "Réactions des ouvriers de l'usine Peugeot Talbot de Poissy à l'annonce du plan social ", JT d'Antenne 2 de 13 heures du 21 juillet 1983. 
celle de Poissy, qui est indéniablement la plus touchée avec une perte supérieure à 4000 emplois, se décomposant en un gros millier de départs en préretraite et presque 3000 licenciements, affectant la CSL s'est, en effet, ralliée à l'arrêt de travail décrété par la CGT et la CFDT. Le slogan «Refusons les licenciements et la mort de Talbot », qui fleurit sur les murs, reflète les craintes syndicales que ces mesures prises par le nouveau PDG, Jacques Calvet, ne soient pas un simple ajustement conjoncturel mais, au contraire, une réorganisation structurelle à l'échelle du groupe qui verrait l'usine de Poissy produire à brève échéance uniquement des véhicules de la gamme Peugeot, voire fermer purement et simplement. Les ouvriers, conscients de ces enjeux, font preuve d'une grande lucidité. Alors que l'un explique que le groupe PSA ne veut plus vendre de Talbot, un autre rappelle que lui et ses camarades sont des "robots vivants " n'ayant bénéficié d'aucune formation pour affronter le chômage et réussir leur reconversion. À près de 40 ans, dont presque la moitié passée en France, il exclut cependant catégoriquement toute idée de retour au pays ${ }^{23}$. Pour la mise en œuvre de ces mesures, l'aval du gouvernement de gauche est néanmoins nécessaire. Alors que les ouvriers partent en vacances, les pouvoirs publics restent, durant de longues semaines, quasiment muets sur le sujet. Cependant, au cœur de l'été, le 3 août sur TF1, le communiste Jack Ralite, ministre délégué chargé de l'Emploi, s'oppose à ce que soit entérinée sans examen la suppression de $10 \%$ des effectifs du groupe PSA et de $26 \%$ de ceux de Poissy. Il annonce la nomination prochaine d'un expert ${ }^{24}$ chargé d'évaluer les difficultés réelles de l'entreprise $^{25}$. Le 12 octobre, le gouvernement accepte les pré-retraites mais refuse les licenciements en l'absence d'un plan social d'accompagnement. Le 21 novembre, la direction fait de nouvelles propositions et un Bureau d'aide à l'orientation et au reclassement du personnel Talbot et Cie, recensant tous les emplois disponibles dans la région, est ouvert. Mais l'inadéquation est grande entre 
les compétences professionnelles des OS immigrés et les emplois proposés, c'est pourquoi une nouvelle grève éclate le 7 décembre à Poissy à l'appel de la CGT et de la CFDT . Le 17 décembre, Jack Ralite est de retour sur le plateau de la première chaîne, quelques heures après que Matignon ait finalement annoncé son accord pour 1905 licenciements chez Talbot, soit les deux tiers de ce que demandait initialement la direction. Devant ce qui pourrait apparaître comme un reniement du gouvernement de gauche converti à une politique de rigueur, le ministre revendique le souci de " traiter humainement les mutations technologiques » et annonce que, grâce aux possibilités de reclassement et de formation professionnelle, il existe un espoir que d'ici un an aucun ouvrier ne soit plus au chômage ${ }^{26}$. Les positions syndicales connaissent elles aussi une sérieuse inflexion, qui se traduit également par l'abandon des OS immigrés. Cela est bien illustré par deux interviews du secrétaire général CGT de la branche métallurgie, réalisées à cinq mois d'intervalle. André Sainjon déclare ainsi, le 19 juillet 1983, que la volonté du groupe PSA de faire disparaître Talbot constitue une "véritable provocation " et que la seule alternative s'offrant aux salariés pour défendre leur outil de travail et leurs emplois est de se mettre en grève avec l'appui de la CGT ${ }^{27}$. Le 21 décembre 1983, il tente au contraire de justifier le ralliement de sa centrale à l'accord trouvé entre le gouvernement et Jacques Calvet. Dès le 17 décembre, les plus hautes instances de la CGT n'ont en effet pas hésité à le qualifier de "compromis acceptable et positif », car son volet industriel prévoit le maintien de la marque Talbot et de ses productions. Du point de vue social, la direction de la CGT a mis de l'eau dans son vin, puisqu'elle ne conteste plus les licenciements, mais demande simplement que les situations soient étudiées cas par cas pour apporter une « réponse positive à chaque travailleur » et lui éviter ainsi le chômage ${ }^{28}$.

\section{Vers un affrontement entre Français et immigrés}

La figure de Nora Tréhel, interviewée à de multiples reprises avec en arrière-plan un groupe compact de travailleurs français et immigrés agitant les drapeaux de la CGT, fait certes télévisuellement contrepoids à cette nouvelle position de la centrale exprimée par André Sainjon assis derrière son bureau en costume cravate. Mais la lutte collective qu'elle personnifie se heurte, en cette fin décembre 1983, à des velléités de sécession de certains OS immigrés, également militants de la CGT, qui ne croient pas à la possibilité de retrouver un emploi en cas de licenciement et souhaitent négocier, hors du syndicat, une aide au retour non plus d'1 million comme en 1977 mais de 20 millions $^{29}$. La lourde porte d'atelier de l'usine Talbot de Poissy qui

En janvier 1984, les affrontements entre Français et immigrés au cœur même de l'usine Talbot de Poissy et leur lot de propos racistes marquent symboliquement une rupture définitive avec l'opinion et l'effacement de la scène médiatique de la génération des pères. se referme devant les caméras pour tenir meeting à huis clos constitue non seulement une interdiction faite aux journalistes de suivre les débats internes, mais symbolise surtout les premiers ferments de division entre travailleurs français et immigrés, d'ailleurs dénoncés par la leader cégétiste dès sa sortie $e^{30}$.

L'occupation de l'usine s'étant poursuivie afin de forcer la direction et le gouvernement à revenir sur leur décision et à négocier, les forces de l’ordre évacuent les grévistes au cours de la nuit de la SaintSylvestre, mais ces derniers ne tardent pas à réinvestir les lieux. Ainsi, dans la matinée du 5 janvier 1984, Talbot Poissy est une nouvelle fois le théâtre de heurts violents qui font 55 blessés, dont 4 dans

26. "Plateau Jack Ralite ", JT de TF1 de 20 heures du 17 décembre 1983. 27. "Conférence de presse Sainjon ", JT de TF1 de 20 heures du 19 juillet 1983. 28. "Interview Sainjon ", JT de TF1 de 13 heures du 21 décembre 1983. 29. 204 000 francs. Voir Vincent Gay, "Lutter pour partir ou pour rester? Licenciements et aide au retour des travailleurs immigrés dans le conflit Talbot 1983-1984", in Travail et emploi, n 137, janvier-mars 2014, pp. 37-50 . 30. "Grève Talbot ", JT d'Antenne 2 de 20 heures du 26 décembre 1983. 
un état grave. Les images de dialogue entre la base et la maîtrise, filmées et mises en scène à Flins douze mois auparavant presque jour pour jour, sont bel et bien révolues. Les militants de la CSL et des personnes extérieures à l'usine attaquent les grévistes retranchés dans le bâtiment $\mathrm{B} 5$, mais cela dépasse de beaucoup le seul enjeu de la reprise du travail. Le reportage diffusé par Antenne 2 dès son édition de la mi-journée montre ainsi parfaitement que ce face-à-face a pris les traits d'un véritable affrontement racial. Lorsqu'un travailleur immigré blessé est évacué, les assaillants crient "Les Noirs au four, les Arabes à la Seine». Puis, revenus sur le parking et désormais retenus par un important cordon de CRS, ils entonnent La Marseillaise tout en continuant à bloquer la sortie des travailleurs immigrés ${ }^{31}$.

Le choc de ces images est d'autant plus grand que le traitement journalistique des différentes grèves, qui se sont succédé depuis 1981, ne s'est pas focalisé sur la question du racisme mais plutôt sur des problématiques sociales communes aux Français et aux immigrés. On demande certes aux OS s'ils constatent dans leur vie quotidienne une augmentation des sentiments de rejet à leur égard, alors que certains ouvriers français expriment leur désir que la priorité soit donnée à leurs propres enfants touchés par le chômage ${ }^{32}$. Mais aucun parallèle systématique n'est établi entre le blocage des usines par des travailleurs immigrés et le climat raciste qui règne en particulier en 1983, comme en témoignent les violences parfois meurtrières contre les « jeunes Maghrébins » dans les cités lors de la période estivale ou le meurtre survenu quelques mois plus tard d'un touriste algérien dans le train Bordeaux-Vintimille. Ces mouvements ne sont pas non plus directement mis en relation avec le premier succès électoral majeur du Front national lors de la municipale partielle de Dreux de septembre 1983.

\section{Quand les fils remplacent les pères}

Ces images d'affrontements et de haine entre Français et immigrés contrastent fortement avec l'accueil réservé un mois plus tôt aux jeunes de la Marche pour l'égalité et contre le racisme, soutenus par des dizaines de milliers de personnes lors de leur arrivée dans la capitale. Sous le feu des caméras, lors des journaux de 20 heures du 3 décembre 1983, une délégation de marcheurs s'entretient avec François Mitterrand à l'Élysée $e^{33}$. Loin de cette apothéose médiatique sous les ors de la République, la rencontre du 16 janvier 1984 entre les marcheurs et les travailleurs en lutte, lors d'une manifestation de soutien aux grévistes de Talbot, fait l'objet d'un traitement télévisuel minimal. Quelques jours plus tôt, le sujet annonçant cet événement est d'ailleurs intitulé «Les Beurs et le retour au pays ${ }^{34}$ ». Après avoir bénéficié d’une visibilité éphémère mais réelle entre fin 1981 et début 1984, la « première génération » disparaît ainsi presque entièrement des écrans dans l'indifférence la plus totale. Dans les reportages consacrés aux OS et à leur éventuel retour au pays, l'attention se focalise parfois, dès l'automne 1983, davantage sur le sort réservé aux jeunes ayant grandi en France que sur celui du père de famille perçu comme inéluctable. Pour bien comprendre ce phénomène, il faut se souvenir que, dans les années 1970, à l'époque du regroupement familial, les médias télévisuels ont clairement opposé, d'une part, les mères immigrées souffrant d'isolement, recluses dans leur appartement, et, d'autre part, leurs enfants qui, par le truchement de l'école de la République, entrent au contraire rapidement en contact avec leurs camarades français après un rapide passage par les classes d'apprentissage de la langue. Moins 
d'une décennie plus tard, leur destinée préoccupe donc toujours les journalistes, comme l'illustre un sujet de plus de quatre minutes diffusé le 9 septembre 1983 dans le journal de la mi-journée d'Antenne 2. Il s'agit en l'occurrence de l'interview par Martine Gilson de Saïd Benami, ouvrier marocain de Talbot Poissy sur lequel pèse la menace d'un licenciement. Ce dernier évoque ses seize années passées en France, pays dont il a contribué à accroître la richesse par son pénible labeur. Il aborde certes la difficulté qui serait la sienne de retrouver un emploi dans l'Hexagone ou dans son pays d'origine car il a déjà 40 ans. Mais il rappelle surtout que le véritable " problème " qui préoccupe les travailleurs immigrés, c'est le devenir de leurs enfants. Éduqués sur les mêmes bancs que leurs camarades français souvent depuis la maternelle, ils seraient incapables, à l'instar de sa fille de 11 ans ou de son fils de 9 ans, de s'adapter à une société marocaine dont ils ignorent presque tout, ne maîtrisant même pas l'arabe. La teneur des propos est visuellement renforcée par le cadre choisi pour l'interview, c'est-à-dire le salon familial, où derrière le père assis sur le canapé on aperçoit son fils et deux camarades ${ }^{35}$. Tout se passe comme si le protagoniste principal tentait de faire abstraction de son propre sort et consentait à se sacrifier la mort dans lâme, mais ne pouvait se résoudre à ce que ses enfants subissent les conséquences d'un retour, qui pour eux n'en est pas un.

\section{Conclusion}

Dans un contexte de crise et de restructuration de l'industrie automobile se traduisant par d'importantes diminutions d'effectifs, le traitement médiatique des grèves du début de la décennie 1980 met en exergue les revendications de dignité et d'égalité des OS immigrés muselés et humiliés depuis tant d'années. Ce qui semble alors en marche, c'est non seulement l'inclusion de ces individus au sein de la classe ouvrière, bien que celle-ci soit en voie de passer du statut de contre-culture à celui de sous-culture. Mais aussi, plus généralement, leur intégration à une société française dans laquelle leurs enfants ont grandi. Cependant, l’année 1983 est annonciatrice de stigmatisations présentes et futures à l'égard de cette "première génération » mais aussi de leurs enfants. En contradiction totale avec les espoirs suscités lors de lélection de François Mitterrand, le gouvernement socialiste tente dès janvier de discréditer ces luttes sociales en les réduisant à une grève de musulmans intégristes manipulés, argumentation à laquelle les médias ne sont néanmoins pas encore pleinement réactifs. Les reportages diffusés dans la seconde partie de l'année 1983 mettent parfaitement en exergue, d'une part, le fait que le tournant de la rigueur scelle définitivement l'abandon, par une gauche devenue pragmatique, des OS immigrés à leur triste sort et, d'autre part, les choix ambigus effectués par la CGT pour laquelle la défense des intérêts de ces prolétaires venus d'ailleurs ne constitue pas une priorité absolue. En janvier 1984, les affrontements entre Français et immigrés au cœur même de l'usine Talbot de Poissy et leur lot de propos racistes marquent symboliquement une rupture définitive avec l'opinion et l'effacement de la scène médiatique de la génération des pères. Ces derniers ne semblent alors avoir le choix qu'entre être les victimes expiatoires d'un chômage qui monte inexorablement et le retour au pays. 